Heatley, N. G., Kelly, B. K. \& Smith, N. (1952). J. gen. Microbiol. 6, 30-40.

\title{
The Assay of Micrococcin, an almost Insoluble Antibiotic
}

\author{
By N. G. HEATLEY, B. K. KELLY AND NANCY SMITH \\ Sir William Dunn School of Pathology, University of Oxford, and Antibiotics \\ Research Station (Medical Research Council), Clevedon, Somerset
}

SUMMARY: The difficulties of assaying micrococcin by the usual microbiological methods and means of obviating or decreasing some of them are described. Certain detergents have a solubilizing effect on micrococcin and it is possible to assay such 'solutions' by hole-plate or other diffusion methods which cannot otherwise be applied. Low results are obtained by this method in crude fermentation liquors, probably because some of the micrococcin is present in a bound form. Ethanolic solutions of micrococcin have a powerful purple fluorescence in ultraviolet light by which amounts of the order of $2.5 \mu \mathrm{g}$. $/ \mathrm{ml}$. may be accurately estimated. Details are given of the procedure, and of the special precautions necessary when micrococcin in broth or tissue is to be estimated. Micrococcin may also be estimated by measuring the absorption in ethanolic solution at $345 \mathrm{~m} \mu$., at which wavelength $E_{1 \mathrm{~cm} .}^{\mathrm{1}}=\mathbf{1 7 7 \cdot 5}$.

Micrococcin was first described by Su (1948), who investigated several microbiological methods for its assay. These are complicated by (1) the low solubility of micrococcin in water (of the order of $1 \mu \mathrm{g} . / \mathrm{ml}$.), (2) the ease with which bacteria become resistant to it, and (3) its relatively high molecular weight. The present paper describes further work on microbiological methods (mainly at Clevedon) and on physical methods (at Oxford); a third section describes the use of detergents in the diffusion assay of micrococcin, a technique which may be applicable to other relatively insoluble antibiotics. Pure micrococcin (Heatley \& Doery, 1951) was used throughout, except in the experiments with crude micrococcin-containing culture fluid.

\section{GENERAL MICROBIOLOGICAL METHODS OF ASSAY}

Diffusion methods. The paper disk, hole-plate, cylinder-plate and similar methods are unreliable, because a workable zone diameter is only given by supersaturated solutions; the conditions governing the degree and duration of supersaturation are obscure. In addition, the curve relating zone diameter to concentration is unusually flat, each doubling of concentration resulting in an increment of 0.6-0.9 mm. in the zone diameter. Nevertheless, Su (1948) found the method useful for semi-quantitative assay of relatively low potency culture fluids.

The direct diffusion assay of micrococcin in organic solvents by dipping a paper disk into the solution and allowing it to dry before placing on a seeded plate was also reported by Su. Although it has given good results with some antibiotics, this method has since been found to be particularly unreliable for micrococcin.

Dilution methods. Since suitable test organisms are inhibited by concentra- 
tions of micrococcin well below that in a saturated watery solution ( $<1 \mu \mathrm{g} . / \mathrm{ml}$.), the first difficulty mentioned for diffusion assay does not arise. Su showed, however, and it has since been confirmed, that resistant mutants are readily formed in a single step or may be present initially, so that dilution tests in fluid medium are unreliable.

The solid medium dilution test ( $\mathrm{Su}, 1948)$ is more satisfactory and is still perhaps the method of choice. Double strength nutrient agar was mixed with serial dilutions in water of the sample to be assayed. The plugged tubes were autoclaved, mixed, and allowed to cool in an inclined position. The surface of each slope was then flooded with a suspension of spores of the test organism and the excess inoculum drained by inverting the tube in a large test-tube where it was left during overnight incubation at $37^{\circ}$. In his later work $\mathrm{Su}$ covered the whole rack, containing up to eighty tubes, with a metal tray and inverted it as a unit. In using Su's method the test organism he specified (Bacillus subtilis, G 8B) was not available, but satisfactory results have been obtained with either of the following inocula:

(1) One or two drops of a 4-6 hr. broth culture of ' $B$. subtilis' NCTC 6346. This culture, which was almost certainly a strain of $B$. cereus (K. Crawford, personal communication), was usually inhibited by $0 \cdot 03-0 \cdot 1 \mu \mathrm{g}$. micrococcin $/ \mathrm{ml}$.

(2) One-fifth of a millilitre of a spore suspension of $B$. subtilis 288 , added from an automatic pipette. The organism was grown for 10-16 days on a Difco yeast beef agar slope and the growth (mostly spores) washed off and diluted to match Brown's opacity tube no. 2 (Burroughs Wellcome and Co. Ltd). This suspension, which could be stored almost indefinitely, was further diluted 16 times before use. This organism was inhibited by $0 \cdot 04 \mu \mathrm{g}$. micrococcin $/ \mathrm{ml}$. The end-point was almost independent of the starting $\mathrm{pH}$ within the range pH 6-8.

The end-point was compared with that in a control series containing known concentrations of micrococcin. A sharp end-point was usually obtained with dilution steps differing by $25 \%$, especially when Evans's peptone was used in the nutrient agar.

When solutions of micrococcin in ethanol or other organic solvents were to be assayed it was necessary to ensure that the concentration of solvent in the first tube was low enough not to interfere. (The titre may be altered by concentrations of solvent well below that which interferes with the growth of the test organism in the absence of micrococcin.) With ethanol, for example, the final concentration must not exceed $1 \%$. When the solution to be assayed could not be diluted enough to achieve this, a portion was evaporated to dryness, taken up in a few drops of warm ethanol, then made up to volume with water or buffer. One advantage of the fact, made use of in Su's method, that micrococcin is sufficiently stable to withstand autoclaving, is that for much of the setting-up of the test an aseptic technique is not necessary. On the other hand, bulk inoculation is ruled out.

Solid medium dilution methods with other organisms and other end-points, such as the change in colour of a $\mathrm{pH}$ or oxidation-reduction indicator, have none of them proved as satisfactory as the method outlined above. However, 
the reduction of methylene blue in a milk medium, as worked out for other antibiotics (e.g. Reid \& Brewer, 1946; Hirsch, 1950) gives results with micrococcin, using Strep. cremoris as test organism, within about $2 \mathrm{hr}$. In some cases the speed with which results are obtained may offset the relative broadness of the end-point.

In an attempt to simplify the dilution procedure, known volumes of serial dilutions were placed in holes in a seeded plate which was incubated. The end-point, taken as the highest dilution which just failed to give perceptible inhibition, was much less sharp than that by Su's method; this modification was therefore abandoned. The same principle had been tried by Su (unpublished), using paper disks or perforated paper disks. It had been hoped that with the latter there would be a sharp all-or-none growth on the surface of the agar exposed by the perforation, since the antibiotic would be diffusing to it in a convergent fashion. Here, too, the results were disappointing.

Lag phase method. This method, developed for the assay of nisin by Hirsch (1950), was tested with micrococcin. The lag phase of Staphylococcus aureus and Strep. faecalis was prolonged, and with the latter organism a linear relationship occurred between $\mathrm{pH}$ and $\log$ of micrococcin concentration over the range $0 \cdot 03-0 \cdot 1 \mu \mathrm{g} . / \mathrm{ml}$. This range was felt to be too narrow and the method was not pursued.

Turbidimetric method. A few trial experiments gave promising results. The medium consisted of: Evans's peptone $1 \%$, Marmite $0.15 \%$, Lemco 0.3 \%, $\mathrm{NaCl} \mathbf{0 . 3 5} \%$, and glucose $0.1 \%$, adjusted to $\mathrm{pH} \mathbf{7 \cdot 4}$ before sterilizing. Three strains of staphylococci were tested, strain $209 \mathrm{P}$ being selected as the most suitable. A range of dilutions of micrococcin in sterile water, each $2 \mathrm{ml}$. in volume in a screw-capped phial, was prepared. To each phial was added $18 \mathrm{ml}$. of the above medium inoculated with one-twentieth of its volume of a $16 \mathrm{hr}$. broth culture of the staphylococcus. After $4 \frac{1}{2} \mathrm{hr}$. at $37^{\circ}$ the turbidities were measured by a Spekker absorptiometer. Typical results are shown in Fig. 1.

The effect of varying some of the conditions (e.g. time of incubation, initial $\mathrm{pH}$ value) was next examined. The effect of different incubation times is shown in Fig. 2. When a smaller inoculum was used similar results were obtained, but the turbidity at the end of $4 \frac{1}{2} \mathrm{hr}$. was somewhat less. Decreasing the glucose concentration to $0.05 \%$ instead of $0.1 \%$ also diminished the turbidity. It seems likely that satisfactory results could be obtained under a variety of conditions provided an alteration in one factor were balanced by appropriate adjustment of the others. Although this method was not used (since the $\mathrm{Su}$ modification was running smoothly) it would seem to have distinct possibilities; results are obtained in a few hours, the method is sensitive, and aseptic conditions are relatively unimportant.

\section{THE DIFFUSION ASSAY OF MICROCOCCIN IN THE PRESENCE OF DETERGENTS}

The solubilizing effect of bile and several synthetic detergents on micrococcin (Heatley, Gowans, Florey \& Sanders, 1952) suggested the possibility of assaying such 'solutions' by a diffusion method. Detergents have been used in the 
diffusion assay of polymixin (Stansly \& Schlosser, 1947) and nisin (Friedmann \& Beach, 1951), but the intention in those cases seems to have been to facilitate diffusion rather than to increase solubility.

Preliminary experiments were made with Triton WR-1339 (a product of Rohm and Haas Co., kindly supplied by Messrs Charles Lennig and Co.

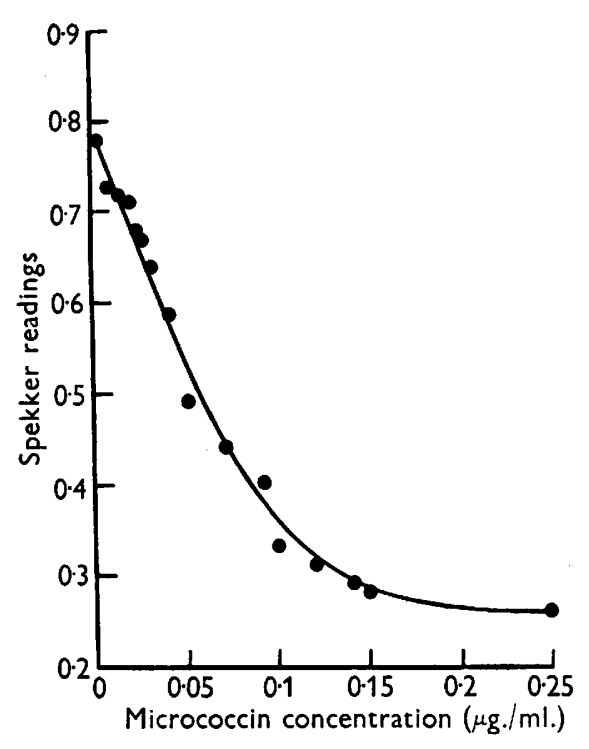

Fig. 1

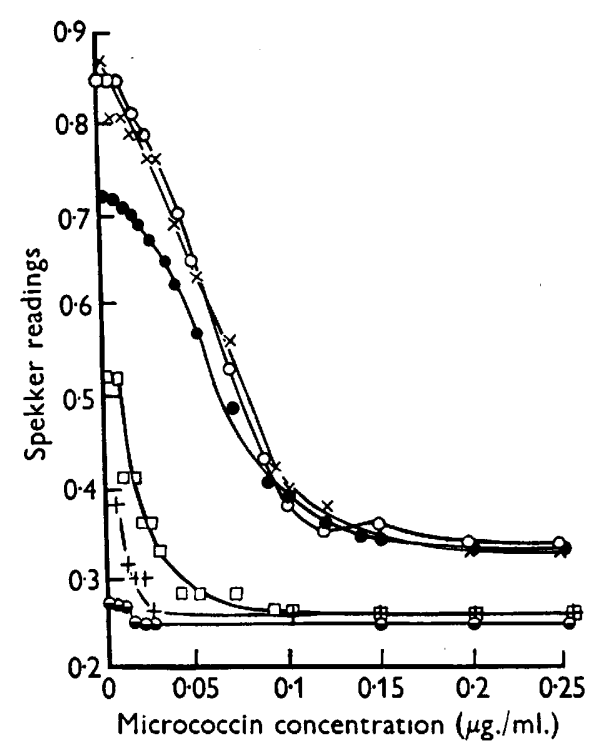

Fig. 2

Fig. 1. The effect of micrococcin concentration on growth (measured turbidimetrically) of Staph. aureus $209 \mathrm{P}$ after $4 \frac{1}{2} \mathrm{hr}$. incubation at $37^{\circ}$.

Fig. 2. The effect of micrococcin concentration on growth (measured turbidimetrically) of Staph. aureus $209 \mathrm{P}$ incubated at $37^{\circ}$ for varying lengths of time: $\bullet-\Theta$, $1 \mathrm{hr}$.; $+\longrightarrow+, 2 \mathrm{hr} . ; \square-\square, 3 \mathrm{hr} . ; \bigcirc-, 4 \mathrm{hr} . ; \times \longrightarrow \times, 5 \mathrm{hr} . ; 0-0,6 \mathrm{hr}$.

[Great Britain] Ltd., 18-20 York Buildings, Adelphi, London, W.C. 2), the non-ionic detergent used by Heatley et al. (1952). Even high concentrations did not interfere with the growth of the test organisms. However, it profoundly depressed the antibacterial activity of micrococcin; thus, the minimum inhibitory concentration of the latter to $B$. subtilis was increased $\mathbf{3 0}$-fold by $0.1 \%$ Triton and more than 125 -fold by $0.9 \%$ Triton. As a result, one effect of the detergent in diffusion assays with very low concentrations of micrococcin was to decrease the size of the inhibition zones. The second effect, resulting from the solubilization of the micrococcin, was to permit, with higher concentrations of the antibiotic, zones larger than the small maximum size obtained in its absence. These points are illustrated in Fig. 3, which shows that as the detergent concentration was increased, the plot of zone diameter against log concentration became linear over a longer range; on the other hand, the higher the concentration of detergent, the smaller was the zone for any given micrococcin concentration (though the decrease in zone diameter was much less than might be expected from the results of dilution tests). When 
detergent was incorporated in the solid medium instead of, or as well as, in the solution in the cylinder, the diminution of the zone size was accentuated; for the main part of the work, therefore, detergent was added only to the solutions to be assayed. Besides Triton WR-1339 (and Triton A-20, which is a solution of it) the following detergents solubilized micrococcin: Crill 10, Tween 80, Teepol, Gemex-X, Aerosol OT, and Tergitol. The last three were strongly inhibitory to $B$. subtilis (the routine test organism) and were not used further.

Degree of curvature and slope of response curves under different conditions. After preliminary experiments the selected detergents were tested in varying concentrations with three concentrations of micrococcin, usually 200,40 and $8 \mu \mathrm{g} . / \mathrm{ml}$., or half these concentrations, in all possible combinations. The mean zone diameter of eight replicates on fifteen large plates (prepared as described below) in an incomplete block design was used to calculate slope and degree of curvature.

Table 1. The degree of curvature and slopes obtained for solutions of micrococcin in different concentrations of various detergents

\begin{tabular}{|c|c|c|c|}
\hline Detergent & $\begin{array}{c}\text { Concentration } \\
\text { in phosphate } \\
\text { buffer (pH 8.0) } \\
(\%)\end{array}$ & $\begin{array}{l}\text { Degree of } \\
\text { curvature }\end{array}$ & Slope \\
\hline Tween 80 & $\begin{array}{l}0 \\
0.5 \\
1 \cdot 0 \\
1 \cdot 5 \\
2 \cdot 0\end{array}$ & $\begin{array}{l}+1 \cdot 6 \\
-1 \cdot 01 \\
-2 \cdot 84 \\
-16 \cdot 38 \\
-13 \cdot 48\end{array}$ & $\begin{array}{r}1 \cdot 68 \\
21 \cdot 95 \\
23 \cdot 24 \\
38 \cdot 28 \\
35 \cdot 84\end{array}$ \\
\hline Crill 10 & $\begin{array}{l}0.6 \\
0 \cdot 8 \\
1 \cdot 0 \\
1 \cdot 2 \\
1 \cdot 4\end{array}$ & $\begin{array}{l}-3 \cdot 81 \\
+0 \cdot 23 \\
+0 \cdot 61 \\
+0 \cdot 09 \\
+0 \cdot 14\end{array}$ & $\begin{array}{l}18 \cdot 57 \\
19 \cdot 93 \\
21 \cdot 05 \\
21 \cdot 59 \\
22 \cdot 12\end{array}$ \\
\hline Triton WR-1339 & $\begin{array}{l}1 \cdot 0 \\
1 \cdot 05 \\
1 \cdot 1 \\
1 \cdot 15 \\
1 \cdot 2\end{array}$ & $\begin{array}{l}-1 \cdot 48 \\
-2 \cdot 18 \\
-1 \cdot 76 \\
-1 \cdot 4 \\
-1 \cdot 52\end{array}$ & $\begin{array}{l}18 \cdot 96 \\
19 \cdot 50 \\
19 \cdot 50 \\
19 \cdot 64 \\
19 \cdot 66\end{array}$ \\
\hline Teepol & $\begin{array}{l}1 \\
2 \\
4\end{array}$ & $\begin{array}{r}-6.61 \\
-1.63 \\
+1.08\end{array}$ & $\begin{array}{l}14 \cdot 29 \\
15 \cdot 63 \\
16 \cdot 94\end{array}$ \\
\hline
\end{tabular}

The degree of curvature was taken as the sum of the mean responses for the high and low micrococcin concentrations minus twice the mean of that for the middle concentration. Slope was calculated from the mean responses for the high and low concentrations.

The results, summarized in Table 1 , show that there is little to choose between the detergents. However, Tween 80 produced readable zones with lower concentrations of micrococcin than did the other detergents, so this compound, at a final concentration of $0.5 \%$, was selected for routine use.

Linearity tests. With the selected concentrations of detergents eight concentrations of micrococcin ranging from 200 to $7 \cdot 5 \mu \mathrm{g} . / \mathrm{ml}$. or from 100 to $4 \mu \mathrm{g} . / \mathrm{ml}$. were set up to determine whether the log concentration-zone diameter 
plot was linear. In every case significant second degree curvature was found, but no higher degree terms were significant. It has been shown (Gridgeman, 1943; Wood, 1944) that when a four-point assay technique is employed, second degree curvature does not affect the estimate of potency ratio.

\section{Routine procedure}

Preparation of seeded plates. The preparation of the large assay plates was as described by Brownlee et al. (1948), except that each $110 \mathrm{ml}$. of melted medium was inoculated with $1 \mathrm{ml}$. of a suspension (diluted to match Brown's opacity tube no. 1) in distilled water, of a 10-14 day slant of B. subtilis 288.

Preparation of standard and unknown solutions. The final concentration of Tween 80 was in all cases $0.5 \%$. The two standard solutions contained 20 and $4 \mu \mathrm{g} . / \mathrm{ml}$. and were prepared by diluting with buffer (and Tween) a stock ethanolic solution containing $2 \mathrm{mg}$. micrococcin $/ \mathrm{ml}$. The unknowns were diluted to about the same strength, the low concentration of unknown being exactly one-fifth that in the high concentration. It was felt to be an advantage to leave the first dilutions containing Tween at room temperature for some time, for solubilization of micrococcin to occur, before making the final dilutions. Ethanolic solutions containing less than $2 \mathrm{mg}$. micrococcin $/ \mathrm{ml}$. or solutions in other solvents were evaporated to dryness in a current of warm air, taken up in a few drops of ethanol, then diluted with the appropriate volume of $0.5 \%$ Tween.

Temperature of incubation, etc. The plates were incubated overnight at $32^{\circ}$. They were read in the projection apparatus of Brownlee et al. (1948).

Reproducibility. Some indication of this is given by the results of two sets of assays of the standard against itself:

(1) The ethanolic standard solution was diluted with Tween 80 solution to give a final concentration of $20 \mu \mathrm{g}$. micrococcin $/ \mathrm{ml}$. in a Tween concentration of $0.5 \%$. Nine assays of this solution gave a mean apparent content of $19.53 \mu \mathrm{g} . / \mathrm{ml}$., with a standard deviation of 1.50 .

(2) The ethanolic standard solution was diluted 1/100 with ethanol. A portion of this was evaporated to dryness and taken up in $0.5 \%$ Tween as described above, to give a solution containing $20 \mu \mathrm{g}$. micrococcin $/ \mathrm{ml}$. Nine assays of this solution gave a mean apparent content of $21 \cdot 04 \mu \mathrm{g} . / \mathrm{ml}$., with a standard deviation of $0 \cdot 43$.

The increment of zone size is about $1.4 \mathrm{~mm}$. for each doubling of concentration, compared with $1.9 \mathrm{~mm}$. for penicillin or $1.7 \mathrm{~mm}$. for streptomycin, under similar conditions.

\section{Assay of crude culture fluid}

Untreated crude culture fluids of the micrococcus gave much lower titres of micrococcin by the above diffusion method than by Su's dilution method. Simple autoclaving did not correct this, nor did ethanolic extraction of the acid precipitate of the raw broth. However, diffusion assay of an ethanolic extract of the acid precipitate from autoclaved broth gave titres approaching 
those found for the untreated broth by Su's method. Evidently the micrococcin occurs in crude broth in some complex or bound form which does not behave in a diffusion assay in the same way as the extracted material. Clearly the applicability of the diffusion method to crude broth cannot be tested by the usual way of observing the recovery of known amounts of added micrococcin. On the other hand, the yardstick by which this can most safely be tested (the dilution method) is itself very inaccurate. In view of the fact that the need for many broth assays was, at any rate temporarily, past, work on these questions was not pursued, but the result of parallel estimations by the two methods on sixteen different samples of culture fluid is given in Table 2.

Table 2. Parallel estimations on sixteen samples of crude micrococcin-containing broth. (a) By diffusion assay, after ethanol extraction of the acid precipitate of autoclaved samples, $(b)$ by dilution method on solid medium

$\begin{array}{ccc} & \begin{array}{c}\text { Apparent micrococin concentration } \\ (\mu \mathrm{g} . / \mathrm{ml} .)\end{array} \\ \text { Sample } & \begin{array}{c}\text { Diffusion } \\ \text { method }\end{array} & \begin{array}{c}\text { Dilution } \\ \text { method }\end{array} \\ \text { no. } & 15 & 20-28 \\ 1 & 25 & 36-44 \\ 2 & 37 & 44-52 \\ 3 & 14 & 12-20 \\ 4 & 51 & 52-60 \\ 5 & 19 & 20-28 \\ 6 & 38 & 44-52 \\ 7 & 14 & 20-28 \\ 8 & 48 & 44-52 \\ 9 & 14 & 20-28 \\ 10 & 38 & 44-52 \\ 11 & 50 & 52-60 \\ 12 & 64 & 60-68 \\ 13 & 36 & 36-44 \\ 14 & 54 & 60-68 \\ 15 & 51 & 60-68 \\ 16 & & \end{array}$

There remains the question whether the diffusion method is superior to the dilution method for broth assay, in view of the autoclaving, precipitation, washing, etc., which are required. The answer is likely to depend on the personal preferences of the assayist, and also on whether assay plates are in routine use for other purposes and readily available. We feel that there is little to choose between the two methods as regards time consumed, though it is an advantage to have a definite value (by the diffusion method) even though subject to large error, rather than a range of values, as for the dilution method. On the other hand, it must be admitted that the titre given by a dilution method is more fundamental and entails fewer assumptions. Since diffusion methods of assay are purely empirical they should be used with reservation for little known substances, or - as in the present case, perhaps-where the antibiotic may exist in a complex or bound form. 


\section{PHYSICAL METHODS OF ESTIMATION}

\section{Adsorption on alumina}

When a solution of micrococcin in chloroform free from ethanol is passed through a column of Brockmann alumina, the purple fluorescent zone where the micrococcin is adsorbed shows virtually no development when large volumes of fresh ethanol-free chloroform are passed through the column. Thus the volume of the fluorescent zone gives a semi-quantitative measure of the

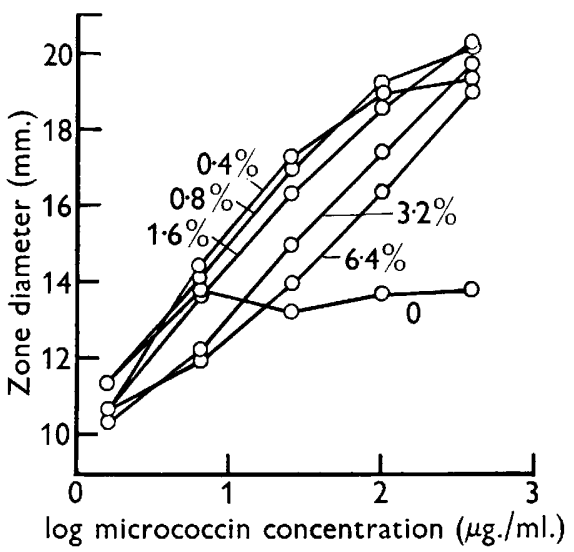

Fig. 3

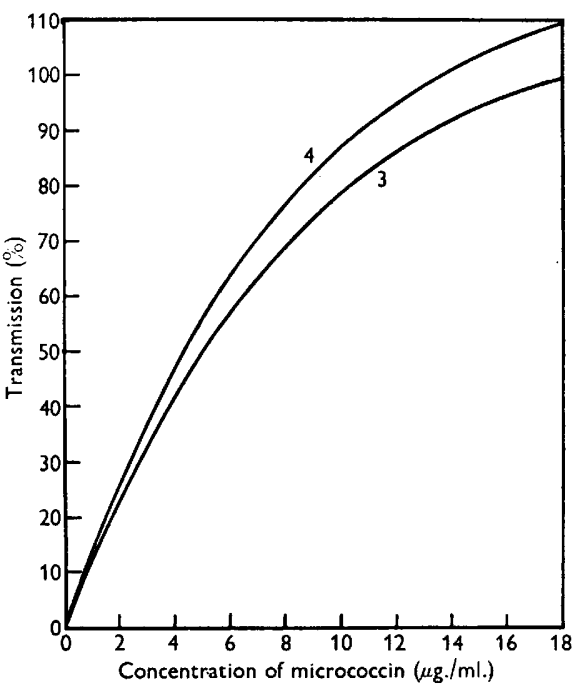

Fig. 4

Fig. 3. The effect on zone diameter of different concentrations of Triton WR-1339 in the diffusion assay (cylinder plate) of micrococcin. Each point is the mean of four zone measurements. Plates bulk-seeded with Staph. aureus NCTC no. 6571. Concentration of Triton as indicated.

Fig. 4. Typical calibration curves relating concentration of micrococcin in ethanol to '\% transmission', as measured on the Beckman model DU spectrophotometer with fluorimeter attachment. Corex cells, $10 \mathrm{~mm}$. Unknowns in position 3 (lower curve) or position 4 (upper curve) of cell holder.

amount of micrococcin present, which is usually $5-15 \mathrm{mg} . / \mathrm{ml}$. of loosely packed alumina. This was not investigated further, but might provide the basis for an assay method similar to that described for aureomycin by Seed \& Wilson (1949).

\section{Fluorimetric estimation}

Micrococcin solutions in ethanol have a powerful purple fluorescence in ultraviolet light. This had early been considered as a method of estimation but was abandoned when a sample submitted for examination in a photographic spectrofluorimeter was reported to have a scarcely detectable fluorescence. However, it was later found that the fluorescence of ethanolic solutions of micrococcin containing $1-15 \mu \mathrm{g} . / \mathrm{ml}$. could be easily and accurately measured in 
the Beckman spectrophotometer, model DU, fitted with the fluorimeter attachment.

It was customary to use plain ethanol in cell $\mathbf{1}$, a standard micrococcin solution containing $5 \mu \mathrm{g} . / \mathrm{ml}$. in cell 2 , and the unknowns in cells 3 and 4 . The sensitivity was adjusted so that the standard solution gave a ' $\%$ transmission' of 50, and the concentration of micrococcin in the unknowns was read off from standard curves (Fig. 4) relating the concentration of micrococcin to $\%$ transmission'. Certain small points, probably well known to users of the Beckman instrument, may be mentioned, as they affected the accuracy of the determinations. (1) The calibration curves for positions 3 and 4 of the cell holder were distinctly different; this was not related to the cell, but to the position (Fig. 4). (2) The cell holder could be moved back and forth within the carrier a few tenths of a millimetre, its actual position determining the value obtained. Attempts to eliminate the play by springs, packing, etc., were not satisfactory, but errors were avoided by always pushing the cell holder against one end of the carrier and moving the latter very cautiously so as not to displace the former. (3) Since the ' $\%$ transmission' approached a maximum with concentrations of micrococcin of the order of $30 \mu \mathrm{g} . / \mathrm{ml}$. and thereafter declined (due to increasing absorption of light by parts of the solution remote from the photocell), a low reading was not necessarily associated with a low concentration of micrococcin. This could be checked at once by visual examination of the solution in ultraviolet light, or by diluting with a little ethanol and observing whether the reading was thereby increased or decreased.

The intensity of fluorescence of any solution is known to vary with several factors, and the fluorimetric estimation of micrococcin has been purely empirical. The fluorescence was not affected by up to $10 \%$ of chloroform, by changes in the concentration of dissolved oxygen, or by acid, though it was much diminished by alkali. Certain tissue extracts, especially hot ethanolic extracts of blood and dilute haemin solutions, had a marked quenching effect. The effect of water was progressively to enhance the fluorescence up to about $60 \%$ of water, beyond which the fluorescence decreased, eventually almost to zero. The magnitude of the effect depended also on the micrococcin concentration (Heatley \& Doery, 1951).

Assay of crude broth by fluorimetry. Ethanolic extracts of broth or other media used for the production of micrococcin (Kelly, Miller \& Hale, 1952) all have a fluorescence equivalent to many times the amount of micrococcin likely to be present; moreover, this non-specific fluorescence may well change during the course of the fermentation, making blank corrections impossible. However, the micrococcin can be freed from much of the non-specific fluorescence by acidifying to $\mathrm{pH} 2$, centrifuging and washing the precipitate with water or dilute acid, suspending it in a few drops of water, adding ethanol to dissolve the micrococcin, and removing residual suspended material by centrifuging. Alternatively, the acid precipitate may be collected on a Hirsch micro funnel, washed in situ with water or dilute acid, and the micrococcin extracted by passing a measured volume of ethanol through the funnel. The accuracy of the estimation is, however, not great because variable amounts 
of non-specific fluorescent material are still present (depending, among other things, on the bulk of the precipitate, which varies greatly), and small amounts of micrococcin are lost in the washing. Several experiments on the estimation of micrococcin in broth by preferential extraction into chloroform gave disappointing results.

The specificity of the fluorimetric assay could probably be increased by restricting the spectral range of the incident light or by measuring the fluorescence over a narrow band of wavelengths, but these proposals could not be tested with the apparatus available.

Assay of tissue extracts by fluorimetry. The natural fluorescence of ethanolic extracts of tissues is much less than that of extracts of culture fluids. When tissue extracts contain high concentrations of micrococcin they can be so much diluted that the fluorescence gives a substantially correct value for micrococcin present. When considerable dilution is not possible, errors arise from two sources (apart from high values obtained when much water is present): (1) fluorescence due to substances other than micrococcin; (2) quenching of the micrococcin fluorescence. In most cases a good correction can be applied for both kinds of error by measuring the fluorescence of similar extracts made from tissues of control animals, alone, and with known concentrations of micrococcin added. It is fortunate that the photo-cell used for measuring the purple fluorescence is not very sensitive to the greenish yellow fluorescence of some, but not all, extracts of intestine, liver and kidney.

\section{Estimation by ultraviolet absorption}

Micrococcin in ethanolic solution has a broad absorption in the ultraviolet with a peak at $345 \mathrm{~m} \mu$, at which Beer's Law is obeyed and $E_{1 \mathrm{~m} .}^{1 \%}=\mathbf{1 7 7 \cdot 5}$. This affords an excellent method for estimating micrococcin; there is no need for a standard and, unlike the fluorescence, the absorption is not changed by other components of the solution. However, the latter may themselves absorb; extracts of broth, for example, do so strongly. For tissue extracts corrections may usually be applied by subtracting the absorption of similar extracts from control animals, but unfortunately extracts prepared in an identical fashion from the same organ of two similar animals sometimes show rather wide differences in absorption.

Our thanks for much helpful assistance are due to Miss E. J. Page, Miss K. E. Payne, Miss I. J. Brodie, Miss M. Hand, Miss H. J. Sulley, Miss E. R. Tompkins, and Miss E. N. Weston.

\section{REFERENCES}

Brownlee, K. A., Delves, C. S., Dorman, M., Green, C. A., Grenfell, E., Jounson, J. D. A. \& Smith, N. (1948). The biological assay of streptomycin by a modified cylinder-plate method. J. gen. Microbiol. 2, 40.

Friedmand, R. \& Beach, S. A. (1951). New methods of assay for the antibiotic nisin. J. gen. Microbiol. 5, v.

Gridgeman, N. T. (1943). The technique of the biological vitamin A assay. Biochem. $J .37,127$.

Heatley, N. G. \& Doery, H. M. (1951). The preparation and some properties of purified micrococcin. Biochem. J. 50, 247. 
Heatley, N. G., Gowans, J. L., Florey, H. W. \& Sanders, A. G. (1952). The effect on experimental tuberculosis and other infections of a Triton-micrococcin mixture. Brit. J. exp. Path. (to be published).

Hirsch, A. (1950). The assay of the antibiotic nisin. J. gen. Microbiol. 4, 70.

Kelly, B. K., Miller, G. A. \& Hale, C. W. (1952). Culture media for largescale production of micrococcin. J. gen. Microbiol. 6, 41.

Reid, R. D. \& Brewer, J. H. (1946). The reductase method for the determination of penicillin concentrations in body fluids. J. Bact. 52, 251.

SEED, J. C. \& Wirson, C. E. (1949). Fluorometric determination of serum aureomycin levels. Science, 110, 707.

Stansly, P. G. \& Schlosser, M. E. (1947). Studies on polymixin: an agar diffusion method of assay. J. Bact. 54, 585 .

Sv, T. L. (1948). Micrococcin. An antibacterial substance formed by a strain of micrococcus. Brit. J. exp. Path. 29, 473.

Woon, E. C. (1944). Mathematics of biological assay. Nature, Lond. 153, 681.

(Received 5 July 1951) 